\title{
Location of Solutions of Fredholm-Nemytskii Integral Equations from a Whittaker-Type Operator
}

\author{
J. A. Ezquerro® and M. A. Hernández-Verón
}

\begin{abstract}
We analyse the global convergence of a Whittaker-type iterative method and obtain restricted global convergence domains, so that we can locate and separate solutions of Fredholm-Nemytskii nonlinear integral equations by means of balls. For this, we use two techniques, one based on the well-known fixed point theorem and the other on a system of recurrence relations. In both techniques, we use the Whittaker-type operator involved and auxiliary functions.
\end{abstract}

Mathematics Subject Classification. 45B05, 47H10, 47H30, 65J15.

Keywords. Whittaker-type operator, fixed point theorem, global convergence, Fredholm integral equation.

\section{Introduction}

We consider Fredholm-Nemytskii integral equations in the form $[2,12,15,17]$

$$
\phi(x)=f(x)+\lambda \int_{a}^{b} \mathcal{N}(x, t) \mathcal{H}(\phi)(t) d t, \quad x \in[a, b], \quad \lambda \in \mathbb{R},
$$

where $f \in \mathcal{C}[a, b]$, the kernel $\mathcal{N}(x, t)$ is a known function in $[a, b] \times[a, b], \mathcal{H}$ is the Nemytskii operator $\mathcal{H}: \mathcal{C}[a, b] \rightarrow \mathcal{C}[a, b]$ such that $\mathcal{H}(\phi)(x)=H(\phi(x))$, where $H: \mathbb{R} \rightarrow \mathbb{R}$, and $\phi(x)$ is the unknown function to be determined.

Generally, we cannot exactly solve integral equations of type (1), so that we can use a numerical method to approximate a solution of the operator $\mathcal{G}: \mathcal{C}[a, b] \rightarrow \mathcal{C}[a, b]$ with

$$
[\mathcal{G}(\phi)](x)=\phi(x)-f(x)-\lambda \int_{a}^{b} \mathcal{N}(x, t) \mathcal{H}(\phi)(t) d t, \quad x \in[a, b], \quad \lambda \in \mathbb{R} .
$$

\footnotetext{
This research was partially supported by Ministerio de Ciencia, Innovación y Universidades under grant PGC2018-095896-B-C21.
} 
It is clear that a solution $\phi^{*}$ of the operator equation (2) is a solution of the integral equation (1). To approximate such a solution we can apply the wellknown method of successive approximations [16], which is known as Picard's method $[6,18]$ when it comes to approximate a solution of the equation $\mathcal{G}(\phi)=$ 0 and defined by $\phi_{n+1}=\phi_{n}-\mathcal{G}\left(\phi_{n}\right), n \geq 0$, with $\phi_{0}$ given in $\mathcal{C}[a, b]$. A generalization of Picard's method when it is applied to a scalar equation $g(t)=0$ is known as Whittaker's method [1,14], given by $t_{n+1}=t_{n}-\mu g\left(t_{n}\right)$, $n \geq 0, \mu \in \mathbb{R}$, with $t_{0}$ given. To solve $\mathcal{G}(\phi)=0$, we follow this generalization and consider a constant operator $A$, such that $A: \mathcal{C}[a, b] \rightarrow \mathcal{C}[a, b]$ with $A(\phi)(x)=k \phi(x)$ and $k \in \mathbb{R}$, and define then the following Whittaker-type iterative method

$$
\phi_{n+1}=W\left(\phi_{n}\right)=\phi_{n}-A \mathcal{G}\left(\phi_{n}\right), \quad n \geq 0, \quad \text { with } \phi_{0} \text { given in } \mathcal{C}[a, b] .
$$

Thus, we are now interested in $\lim _{n} \phi_{n}=\phi^{*}$, where $\phi^{*}$ is a solution of (2) and, therefore, a solution of the integral equation (1). It is well known that the convergence of the sequence $\left\{\phi_{n}\right\}$ can be established in different ways: semilocal convergence, local convergence and global convergence. Global convergence guarantees the convergence of an iterative method starting at any point in a previously located domain and once the existence of a solution of the operator has been proved in the domain. Notice that only conditions on the operator involved are required.

One of the aims of this work is to obtain a result of global convergence for the iterative methods (3). It is well known that the fixed point theorem is a procedure to obtain results of global convergence. So, a fixed point $\phi^{*}$ of the operator

$$
[\mathcal{T}(\phi)](x)=f(x)+\lambda \int_{a}^{b} \mathcal{N}(x, t) \mathcal{H}(\phi)(t) d t, \quad x \in[a, b], \quad \lambda \in \mathbb{R}
$$

is a solution of the equation (1) and we can then use the fixed point theorem to approximate $\phi^{*}$. The fixed point theorem says [3]:

If the operator $\mathcal{T}: \mathcal{C}[a, b] \rightarrow \mathcal{C}[a, b]$ is a contraction, then $\mathcal{T}$ has a unique fixed point $\phi^{*}$ in $\mathcal{C}[a, b]$ that can be approximated by the method of successive approximations, $\phi_{n+1}=\mathcal{T}\left(\phi_{n}\right), n \geq 0$, with $\phi_{0}$ given in $\mathcal{C}[a, b]$.

Remember that the operator $\mathcal{T}$ is a contraction if $\|\mathcal{T}(u)-\mathcal{T}(v)\|<\sigma\|u-v\|$ with $\sigma \in[0,1)$, for all $u, v \in \mathcal{C}[a, b]$. If the operator $\mathcal{T}$ is derivable, it is sufficient that the condition $\left\|\mathcal{T}^{\prime}(u)\right\|<1$, for all $u \in \mathcal{C}[a, b]$, is satisfied to see that $\mathcal{T}$ is a contraction. Then, the method of successive approximations can be applied to approximate a fixed point $\phi^{*}$ of the operator $\mathcal{T}$. However, this result can be applied only if if the operator $\mathcal{T}$ has a unique fixed point $\phi^{*}$ in the full space $\mathcal{C}[a, b]$ and, in addition, it is not necessary to separate it from other possible fixed points.

If we want to consider other possible situations in which the operator $\mathcal{T}$ has more fixed points, we must apply a restricted fixed point theorem, as for example:

If $\mathcal{D}$ is a convex and compact set of $\mathcal{C}[a, b]$ and the operator $\mathcal{T}: \mathcal{D} \rightarrow$ $\mathcal{D}$ is a contraction, then $\mathcal{T}$ has a unique fixed point $\phi^{*}$ in $\mathcal{D}$ that 
can be approximated by the method of successive approximations, $\phi_{n+1}=\mathcal{T}\left(\phi_{n}\right), n \geq 0$, with $\phi_{0}$ given in $\mathcal{D}$.

In this case, the first problem is obviously to locate a domain $\mathcal{D}$ that contains a fixed point of the operator $\mathcal{T}$. For this, we need some information about the possible fixed points of the operator $\mathcal{T}$, which may not be possible in all situations, as we can see in Sect. 2.

As an approximate zero of the operator (2) is a fixed point of the operator $W$ given in (3) (called Whittaker-type operator), we can study the location of fixed points of the operator (4) and the separation between then from the operator $W$. So, the main aim of this study focuses on the qualitative properties of the location of the fixed points of the operator (4) and the separation between them. In Sect. 3, we do this study from the application of the restricted Fixed Point Theorem given above to the operator $W$ and using a technique based on auxiliary points [10-12]. Moreover, we approximate a fixed point $\phi^{*}$ of the operator (4) by the iterative method (3) starting at any function of the considered domain. Therefore, we obtain domains of global convergence for (3), as for the method of successive approximations when the fixed point theorem is applied.

In Sect. 4, we develop also a technique to obtain domains of global convergence that is not based on the fixed point theorem, but on a system of recurrence relations. This technique also uses auxiliary points.

Finally, in Sect. 5, we can find the conclusions of the work.

Throughout the paper, we denote $\overline{B(\phi, r)}=\{\theta \in \mathcal{C}[a, b] ;\|\theta-\phi\| \leq r\}$, $B(\phi, r)=\{y \in \mathcal{C}[a, b] ;\|\theta-\phi\|<r\}$ and the set of bounded linear operators from $\mathcal{C}[a, b]$ to $\mathcal{C}[a, b]$ by $\mathcal{L}(\mathcal{C}[a, b], \mathcal{C}[a, b])$, use the infinity norm in $\mathcal{C}[a, b]$ and illustrate all the theoretical results with examples.

\section{Motivation}

We start by considering a first simple example, where the linear integral equation

$$
\phi(x)=x^{3}+\lambda \int_{0}^{1} \mathrm{e}^{-(x+t)} \phi(t) d t, \quad x \in[0,1], \quad \lambda \in \mathbb{R},
$$

is involved. The corresponding integral operator is then

$$
\left[\mathcal{T}_{1}(\phi)\right](x)=x^{3}+\lambda \int_{0}^{1} \mathrm{e}^{-(x+t)} \phi(t) d t, \quad x \in[0,1], \quad \lambda \in \mathbb{R},
$$

so that

$$
\begin{aligned}
& \left\|\mathcal{T}_{1}(u)-\mathcal{T}_{1}(v)\right\| \leq|\lambda|\left(\int_{0}^{1} \mathrm{e}^{-t} d t\right)\left\|\mathrm{e}^{-x}\right\|\|u-v\| \leq \frac{\mathrm{e}-1}{\mathrm{e}}|\lambda|\|u-v\|, \\
& \quad u, v \in \mathcal{C}[a, b],
\end{aligned}
$$

and $\mathcal{T}_{1}$ is a contraction in $\mathcal{C}[a, b]$ if and only if $|\lambda|<\frac{\mathrm{e}}{\mathrm{e}-1}=1.58198 \ldots$ Then, the integral equation (5) with $|\lambda|<1.58198 \ldots$ has a unique solution $\phi^{*}$ in $\mathcal{C}[a, b]$ that can be approximated by the method of successive approximations, 
$\phi_{n+1}=\mathcal{T}_{1}\left(\phi_{n}\right), n \geq 0$, starting at any $\phi_{0}$ in $\mathcal{C}[a, b]$. For example, if $\lambda=\frac{1}{2}$, then it is easy to check that the solution is $\phi^{*}(x)=x^{3}+(0.0726 \ldots) \mathrm{e}^{-x}$.

Now, we make a change in the linearity of the integral equation (5), choose $\lambda=\frac{1}{5}$ and consider then the following integral equation

$$
\phi(x)=x^{3}+\frac{1}{5} \int_{0}^{1} \mathrm{e}^{-(x+t)} \phi(t)^{2} d t, \quad x \in[0,1],
$$

whose solutions $\phi_{1}(x)=x^{3}+(0.0122 \ldots) \mathrm{e}^{-x}$ and $\phi_{2}(x)=x^{3}+(15.4354 \ldots) \mathrm{e}^{-x}$ are easily calculable. To study the integral equation (6), we take the operator

$$
\left[\mathcal{T}_{2}(\phi)\right](x)=x^{3}+\frac{1}{5} \int_{0}^{1} \mathrm{e}^{-(x+t)} \phi(t)^{2} d t, \quad x \in[0,1] .
$$

As

$$
\left\|\mathcal{T}_{2}(u)-\mathcal{T}_{2}(v)\right\| \leq \frac{1}{2}\left(\int_{0}^{1} \mathrm{e}^{-t} d t\right)\left\|\mathrm{e}^{-x}\right\|(\|u\|+\|v\|)\|u-v\|,
$$

the operator $\mathcal{T}_{2}$ is not a contraction in the full space $\mathcal{C}[a, b]$. To find a convex and compact domain where we can apply the restricted fixed point theorem mentioned above, we locate previously the possible fixed points. For this, if $\phi^{*}$ is a possible fixed point of the operator $\mathcal{T}_{2}$, we have from $(6)$ the following condition

$$
\left\|\phi^{*}\right\| \leq 1+\frac{\mathrm{e}-1}{5 \mathrm{e}}\left\|\phi^{*}\right\|^{2}
$$

which is satisfied if $\left\|\phi^{*}\right\| \leq 1.17435 \ldots$ or $\left\|\phi^{*}\right\| \geq 1.17435 \ldots$ If, for example, we choose $\overline{B(0,2)}$ as the convex and compact set $\mathcal{D}$, then $\left\|\mathcal{T}_{2}(u)-\mathcal{T}_{2}(v)\right\|$ $\leq \frac{4(\mathrm{e}-1)}{5 \mathrm{e}}\|u-v\|$, with $\frac{4(\mathrm{e}-1)}{5 \mathrm{e}}=0.5056 \ldots<1$, and

$$
\left\|\left[\mathcal{T}_{2}(\phi)\right](x)\right\| \leq 1+\frac{\mathrm{e}-1}{5 \mathrm{e}} 2^{2}=1.5057 \ldots<2 .
$$

Therefore, the integral equation (6) has a unique solution $\phi^{*}$ in $\overline{B(0,2)}$ that can be approximated by the method of successive approximations, $\phi_{n+1}=$ $\mathcal{T}_{2}\left(\phi_{n}\right), n \geq 0$, starting at any $\phi_{0}$ in $\overline{B(0,2)}$.

After that, we make a small modification in the above integral by substituting $\lambda=\frac{1}{2}$ for $\lambda=\frac{1}{5}$,

$$
\phi(x)=x^{3}+\frac{1}{2} \int_{0}^{1} \mathrm{e}^{-(x+t)} \phi(t)^{2} d t, \quad x \in[0,1],
$$

so that we take the following integral operator

$$
\left[\mathcal{T}_{3}(\phi)\right](x)=x^{3}+\frac{1}{2} \int_{0}^{1} \mathrm{e}^{-(x+t)} \phi(t)^{2} d t, \quad x \in[0,1],
$$

whose fixed points are $\phi_{1}(x)=x^{3}+(0.0318 \ldots) \mathrm{e}^{-x}$ and $\phi_{2}(x)=x^{3}+$ $(5.9442 \ldots) \mathrm{e}^{-x}$. Obviously, as the previous operator $\mathcal{T}_{2}$, the operator $\mathcal{T}_{3}$ is not a contraction in the full space $\mathcal{C}[0,1]$. Moreover, it is easy to check that it is not possible to locate previously a fixed point. So, we look for a convex and compact set of the form $\overline{B(0, r)}$ in $\mathcal{C}[0,1]$, so that the operator $\mathcal{T}_{3}: \overline{B(0, r)} \rightarrow \overline{B(0, r)}$ is a contraction on it. But, it is easy to check that 
this is not possible. Therefore, there are not domains of the form $\overline{B(0, r)}$ where we can apply the restricted fixed point theorem given above.

As we have just seen, from the restricted fixed point theorem and the pre-location of fixed points, we can sometimes consider finding domains of the form $\overline{B(0, r)}$ that contain a fixed point and separating it from other possible fixed points. However, as we have seen for the last integral equation, it is not always possible to apply this technique. Moreover, it is clear that such domains do not correctly locate a fixed point or separate it from another possible fixed point.

In this work, we consider auxiliary points to be able to locate a fixed point in a domain of the form $\overline{B(\widetilde{\phi}, R)}$, where $\widetilde{\phi}$ is an auxiliary point given in $\mathcal{C}[a, b]$, which allows us to obtain a better location and also separate the fixed point from other possible ones with greater accuracy.

\section{Whittaker-Type Operator}

From the restricted fixed point theorem given in the introduction, we see that the method of successive approximations, $\phi_{n+1}=\mathcal{T}\left(\phi_{n}\right), n \geq 0$, with $\phi_{0}$ given in some $\mathcal{D}$, is convergent if the operator $\mathcal{T}: \mathcal{D} \rightarrow \mathcal{D}$ is a contraction. To prove that $\mathcal{T}$ is a contraction, we can consider that the Nemystkii operator is Lipschitz continuous in some $\mathcal{D} \subset \mathcal{C}[a, b]$,

$$
\|\mathcal{H}(\phi)-\mathcal{H}(\varphi)\| \leq L\|\phi-\varphi\|, \quad \text { for all } \phi, \varphi \in \mathcal{C}[a, b] .
$$

In particular, we consider domains of the form $\mathcal{D}=\overline{B(\widetilde{\phi}, R)}$ and, as we indicate in Sect. 2, an auxiliary function $\widetilde{\phi} \in \mathcal{C}[a, b]$ to locate a fixed point in $\overline{B(\widetilde{\phi}, R)}$ which allows us to obtain a better location and separation of the fixed point. From these two ideas, we establish the following convergence result for iterative method (3), which is also a result on existence and uniqueness of solution.

Theorem 1. Suppose that the Nemystkii operator $\mathcal{H}$ is Lipschitz continuous in $\mathcal{C}[a, b]$, namely $\mathcal{H}$ satisfies the condition $(8)$. Let $\widetilde{\phi} \in \mathcal{C}[a, b]$ and consider $\overline{B(\widetilde{\phi}, R)}$ with

$$
R \geq \frac{|k|\|f-\widetilde{\phi}\|+|\lambda||k| M\|\mathcal{H}(\widetilde{\phi})\|}{1-(|1-k|+|\lambda||k| M L)},
$$

where $M=\left\|\int_{a}^{b} \mathcal{N}(x, t) d t\right\|$ and provided that

$$
|1-k|+|\lambda||k| M L<1 .
$$

Then, the operator $W: \overline{B(\widetilde{\phi}, R)} \rightarrow \overline{B(\widetilde{\phi}, R)}$ has a unique fixed point $\phi^{*}$ and the iterative method (3) starting at any $\phi_{0} \in \overline{B(\widetilde{\phi}, R)}$ converges to $\phi^{*}$.

Proof. First, we prove that $W: \overline{B(\widetilde{\phi}, R)} \rightarrow \overline{B(\widetilde{\phi}, R)}$. If $\phi \in \overline{B(\widetilde{\phi}, R)}$, then

$$
W(\phi)-\widetilde{\phi}=\phi-A \mathcal{G}(\phi)-\widetilde{\phi}=\phi-k \mathcal{G}(\phi)-\widetilde{\phi},
$$


so that

$$
\begin{aligned}
& (W(\phi))(x)-\widetilde{\phi}(x)=(1-k)(\phi(x)-\widetilde{\phi}(x))+k(f(x)-\widetilde{\phi}(x)) \\
& \quad+\lambda k \int_{a}^{b} \mathcal{N}(x, t) \mathcal{H}(\phi)(t) d t .
\end{aligned}
$$

Thus, as $\|\mathcal{H}(\phi)\| \leq\|\mathcal{H}(\widetilde{\phi})\|+L\|\phi-\widetilde{\phi}\|$, we have

$$
\begin{aligned}
\|W(\phi)-\widetilde{\phi}\| & \leq|1-k|\|\phi-\widetilde{\phi}\|+|k|\|f-\widetilde{\phi}\|+|\lambda||k| M\|\mathcal{H}(\phi)\| \\
& \leq(|1-k|+|\lambda||k| M L) R+|k|\|f-\widetilde{\phi}\|+|\lambda||k| M\|\mathcal{H}(\widetilde{\phi})\| .
\end{aligned}
$$

Now, from (9) and (10), it follows that $W(\phi) \in \overline{B(\widetilde{\phi}, R)}$.

Second, from (10), it is easy to see that the operator $W$ is a contraction, since

$\|W(\phi)-W(\varphi)\| \leq(|1-k|+|\lambda||k| M L)\|\phi-\varphi\|, \quad$ for all $\phi, \varphi \in B(\widetilde{\phi}, R)$, and the contractivity factor $\sigma=|1-k|+|\lambda||k| M L$ is such that $\sigma \in[0,1)$.

Third, by applying the restricted fixed point theorem given in the introduction to the operator $W$ in $\overline{B(\widetilde{\phi}, R)}$, the proof is complete.

Next, we illustrate Theorem 1 with the integral equations (6) and (7), seeing that in both cases we can improve the results obtained in Sect. 2.

Example 2. We have seen in Sect. 2 that the integral equation (6) has a unique solution in $\overline{B(0,2)}$. On the other hand, if we apply Theorem 1 with $\widetilde{\phi}(x)=x^{3}$, we have

$$
\|\mathcal{H}(\phi)-\mathcal{H}(\varphi)\| \leq\left\|\phi^{2}-\varphi^{2}\right\| \leq 2(\|\widetilde{\phi}\|+R)\|\phi-\varphi\|=L\|\phi-\varphi\|
$$

where $L=2(\|\widetilde{\phi}\|+R)$. Taking into account that $M=\max _{x \in[0,1]}\left|\int_{0}^{1} \mathrm{e}^{-(x+t)} d t\right|$ $=\frac{\mathrm{e}-1}{\mathrm{e}}=0.6321 \ldots$, we have that the conditions $(9)$ and $(10)$ are satisfied if $k \in(0,1)$ and $R<0.1801 \ldots$ or $k \in[1,1.5963 \ldots)$ and $R<\frac{10 \mathrm{e}+(2-7 \mathrm{e}) k}{2(\mathrm{e}-1) k}$ and $2(\mathrm{e}-1) k R^{2}+(7 \mathrm{e} k-2(5 \mathrm{e}+k)) R+(\mathrm{e}-1) k \leq 0$.

Therefore, from Theorem 1, we can guarantee the existence of a unique solution of the integral equation (6) in $\overline{B(\widetilde{\phi}, R)}=\overline{B\left(x^{3}, R\right)}$ if

$$
R<0.1801 \ldots, \text { provided that } k \in(0,1),
$$

or $R$ is such that

$$
R<\frac{10 \mathrm{e}+(2-7 \mathrm{e}) k}{2(\mathrm{e}-1) k} \text { and } 2(\mathrm{e}-1) k R^{2}+(7 \mathrm{e} k-2(5 \mathrm{e}+k)) R+(\mathrm{e}-1) k \leq 0,
$$

provided that $k \in[1,1.5963 \ldots)$.

Note that the location and separation of a solution of (6) are fixed if $k \in(0,1)$, since the solution is unique in $\overline{B\left(x^{3}, R\right)}$ with $R<0.1801 \ldots$ But, for $k \in[1,1.2254 \ldots)$, it is not. We show in Table 1 some values of 
Table 1. Radii of the balls $\overline{B\left(x^{3}, R\right)}$ where the existence of a unique solution of (6) is guaranteed from Theorem 1 with $R \in\left[R_{1}, R_{2}\right)$ and $k \in[1,1.5963 \ldots)$

\begin{tabular}{llllll}
\hline$k$ & $R_{1}$ & $R_{2}$ & $k$ & $R_{1}$ & $R_{2}$ \\
\hline 1 & $0.1801 \ldots$ & $2.7747 \ldots$ & 1.14 & $0.2963 \ldots$ & $1.6872 \ldots$ \\
1.02 & $0.1917 \ldots$ & $2.6081 \ldots$ & 1.16 & $0.3248 \ldots$ & $1.5390 \ldots$ \\
1.04 & $0.2043 \ldots$ & $2.4463 \ldots$ & 1.18 & $0.3601 \ldots$ & $1.3881 \ldots$ \\
1.06 & $0.2184 \ldots$ & $2.2887 \ldots$ & 1.20 & $0.4064 \ldots$ & $1.2301 \ldots$ \\
1.08 & $0.2342 \ldots$ & $2.1348 \ldots$ & 1.22 & $0.4742 \ldots$ & $1.0543 \ldots$ \\
1.10 & $0.2520 \ldots$ & $1.9838 \ldots$ & 1.24 & $0.6286 \ldots$ & $0.7953 \ldots$ \\
1.12 & $0.2724 \ldots$ & $1.8349 \ldots$ & & & \\
\hline
\end{tabular}

$k \in[1,1.5963 \ldots)$ and the corresponding radii of the balls $\overline{B\left(x^{3}, R\right)}$ with $R \in\left[R_{1}, R_{2}\right)$, where the existence of a unique fixed point is guaranteed and then a solution of the integral equation (6). Observe that the best location of the fixed point, which is in the ball $\overline{B\left(x^{3}, R_{1}\right)}$, and the best separation of other possible ones, which is in the ball $\overline{B\left(x^{3}, R_{2}\right)}$, are better the closer $k$ is to 1 .

If we choose the most favorable situation, which is $k=1$, we obtain that the existence domain of solution is $\overline{B\left(x^{3}, 0.1801 \ldots\right)}$ and the uniqueness domain of solution is $\overline{B\left(x^{3}, 2.7747 \ldots\right)}$, so that we considerably improve the domains obtained by means of the restricted fixed point theorem, since it is $\overline{B(0,2)}$ in both cases, as can be seen in the Figs. 1, 2 .

Example 3. If we consider the integral equation (7), we cannot previously locate a solution. We cannot find either a convex and compact domain of the form $\overline{B(0, r)}$ in $\mathcal{C}[0,1]$ in which $\mathcal{T}_{3}$ is a contraction in such a way that we could apply the restricted fixed point theorem given in the introduction. However, we can locate a solution of (7) by Theorem 1. For this, we choose the auxiliary function $\widetilde{\phi}(x)=x^{3}$. In addition,

$$
\|\mathcal{H}(\phi)-\mathcal{H}(\varphi)\| \leq L\|\phi-\varphi\| \text { with } L=2(\|\widetilde{\phi}\|+R) .
$$

As $M=\frac{\mathrm{e}-1}{\mathrm{e}}=0.6321 \ldots$, the conditions (9) and (10) are satisfied if $k \in(0,1)$ and $R<\frac{1}{\mathrm{e}-1}=0.5819 \ldots$ or $k \in[1,1.2254 \ldots)$ and $R<\frac{2 \mathrm{e}+(1-2 \mathrm{e}) k}{(\mathrm{e}-1) k}$ and $(\mathrm{e}-1) k R^{2}+((2 \mathrm{e}-1) k-4 \mathrm{e}) R+(\mathrm{e}-1) k \leq 0$.

Therefore, we can guarantee the existence of a unique solution of the integral equation (7) in $\overline{B(\widetilde{\phi}, R)}=\overline{B\left(x^{3}, R\right)}$ by Theorem 1 if

$$
R<0.5819 \ldots \text {, provided that } k \in(0,1),
$$

or $R$ is such that

$R<\frac{2 \mathrm{e}+(1-2 \mathrm{e}) k}{(\mathrm{e}-1) k}$ and $(\mathrm{e}-1) k R^{2}+((2 \mathrm{e}-1) k-4 \mathrm{e}) R+(\mathrm{e}-1) k \leq 0$,

provided that $k \in[1,1.2254 \ldots)$. 


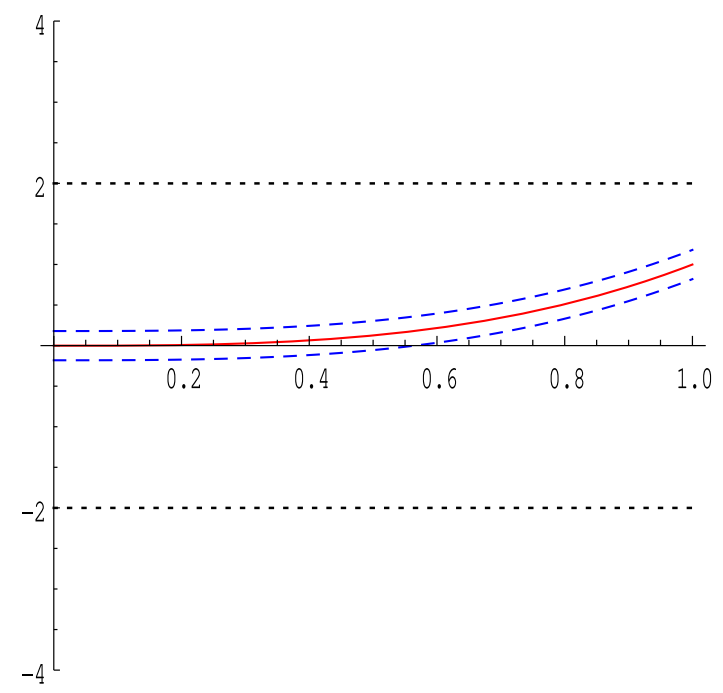

Figure 1. The existence domains of solution $\overline{B(0,2)}$ and $\overline{B\left(x^{3}, 0.1801 \ldots\right)}$ obtained respectively by the restricted fixed point theorem and Theorem 1 for the integral equation (6). The first domain is traced by the dotted line and the second one by the dashed line.

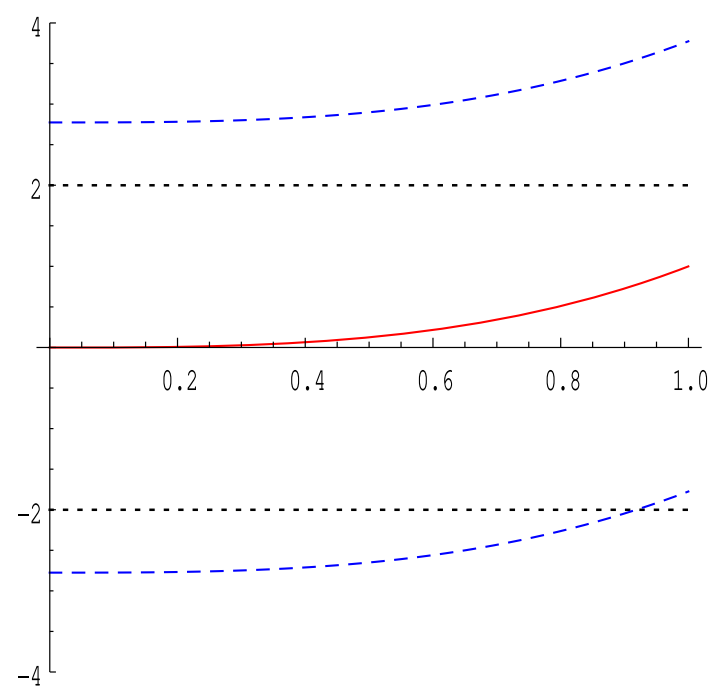

Figure 2. The uniqueness domains of solution $\overline{B(0,2)}$ and $\overline{B\left(x^{3}, 2.7747 \ldots\right)}$ obtained, respectively, by the restricted fixed point theorem and Theorem 1 for the integral equation (6). The first domain is traced by the dotted line and the second one by the dashed line. 
Table 2. Radii of the balls $\overline{B\left(x^{3}, R\right)}$ where the existence of a unique solution of $(7)$ is guaranteed from Theorem 1 with $R \in\left[R_{1}, R_{2}\right)$ and $k \in[1,1.2254 \ldots)$

\begin{tabular}{lll}
\hline$k$ & $R_{1}$ & $R_{2}$ \\
\hline 1 & $0.2892 \ldots$ & $0.5819 \ldots$ \\
1.02 & $0.3011 \ldots$ & $0.5199 \ldots$ \\
1.04 & $0.3135 \ldots$ & $0.4602 \ldots$ \\
1.06 & $0.3266 \ldots$ & $0.4028 \ldots$ \\
1.08 & $0.3405 \ldots$ & $0.3476 \ldots$ \\
\hline
\end{tabular}

Note that the location and separation of a solution of $(7)$ are fixed if $k \in(0,1)$, since the solution is unique in $\overline{B\left(x^{3}, R\right)}$ with $R<0.5819 \ldots$ But, for $k \in[1,1.2254 \ldots)$, it is not. We use in Table 2 some values of $k \in[1,1.2254 \ldots)$ and the corresponding radii of the balls $\overline{B\left(x^{3}, R\right)}$ with $R \in\left[R_{1}, R_{2}\right)$, where the existence of a unique fixed point is guaranteed and then a solution of the integral equation (7). Observe that the best location of the fixed point, which is in the ball $\overline{B\left(x^{3}, R_{1}\right)}$, and the best separation of other possible ones, which is in the ball $\overline{B\left(x^{3}, R_{2}\right)}$, are better the closer $k$ is to 1 .

On the other hand, we know that the operator $\mathcal{T}$ is also a contraction if $\mathcal{T}$ is derivable and such that $\left\|\mathcal{T}^{\prime}(u)\right\|<1$, for all $u \in \mathcal{C}[a, b]$. Taking into account this fact, we can consider that the derivative of Nemytskii operator $\mathcal{H}$ satisfies

$$
\left\|\mathcal{H}^{\prime}(\phi)\right\| \leq \omega_{1}(\|\phi\|), \quad \text { for all } \phi \in \mathcal{C}[a, b],
$$

where $\omega_{1}:[0,+\infty) \rightarrow \mathbb{R}$ is a nondecreasing continuous function such that $\omega_{1}(0) \geq 0([4,7,13])$, or, once $\widetilde{\phi} \in \mathcal{C}[a, b]$ is fixed,

$$
\left\|\mathcal{H}^{\prime}(\phi)-\mathcal{H}^{\prime}(\widetilde{\phi})\right\| \leq \omega_{2}(\|\phi-\widetilde{\phi}\|), \quad \text { for all } \phi \in \mathcal{C}[a, b],
$$

where $\omega_{2}:[0,+\infty) \rightarrow \mathbb{R}$ is a nondecreasing continuous function such that $\omega_{2}(0)=0([4,8,9])$. Under both conditions for $\mathcal{H}^{\prime}$, we can prove that the operator $\mathcal{T}$ is a contraction, as we can see in the following result.

We first observe that

$$
\left[W^{\prime}(\phi) \varphi\right](x)=(I-A) \varphi(x)+\lambda A \int_{a}^{b} \mathcal{N}(x, t)\left[\mathcal{H}^{\prime}(\phi) \varphi\right](t) d t,
$$

since $\left[W^{\prime}(\phi) \varphi\right](x)=\left[\left(I-A \mathcal{G}^{\prime}(\phi)\right) \varphi\right](x)$, where $I$ is the identity operator and $\left[\mathcal{H}^{\prime}(\phi) \varphi\right](t)=H^{\prime}(\phi(t)) \varphi(t)$. And second, establish the following result by applying the restrictive fixed point theorem given in the introduction.

Theorem 4. Let $\widetilde{\phi} \in \mathcal{C}[a, b]$.

(a) Under the condition (11), suppose $R>0$ such that

$$
|k|\|f-\widetilde{\phi}\|+|\lambda||k| M\left(\|\mathcal{H}(\widetilde{\phi})\|+\omega_{1}(\|\widetilde{\phi}\|+R) R\right)+(|1-k|-1) R \leq 0 .
$$


(b) Suppose that there exists $R>0$ such that condition (12) is satisfied in $\mathcal{D}=\overline{B(\widetilde{\phi}, R)}$ and

$|k|\|f-\widetilde{\phi}\|+|\lambda||k| M\left(\|\mathcal{H}(\widetilde{\phi})\|+\left(\left\|\mathcal{H}^{\prime}(\widetilde{\phi})\right\|+\omega_{2}(R)\right) R\right)+(|1-k|-1) R \leq 0$.

Then, the operator $W: \overline{B(\widetilde{\phi}, R)} \rightarrow \overline{B(\widetilde{\phi}, R)}$ has a unique fixed point $\phi^{*}$ and the iterative method (3) starting at any $\phi_{0} \in \overline{B(\widetilde{\phi}, R)}$ converges linearly to $\phi^{*}$.

Proof. Prove item (a). From

$$
\begin{aligned}
& W(\phi)(x)-\widetilde{\phi}(x)=(1-k)(\phi(x)-\widetilde{\phi}(x))+k(f(x)-\widetilde{\phi}(x)) \\
& \quad+\lambda k \int_{a}^{b} \mathcal{N}(x, t) \mathcal{H}(\phi)(t) d t
\end{aligned}
$$

it follows that

$$
\|W(\phi)-\widetilde{\phi}\| \leq|1-k|\|\phi(x)-\widetilde{\phi}(x)\|+|k|\|f(x)-\widetilde{\phi}(x)\|+|\lambda||k| M\|\mathcal{H}(\phi)\| .
$$

Now, from (11), we have

$$
\|\mathcal{H}(\phi)\| \leq\|\mathcal{H}(\phi)-\mathcal{H}(\widetilde{\phi})\|+\|\mathcal{H}(\widetilde{\phi})\| \leq\|\mathcal{H}(\widetilde{\phi})\|+\omega_{1}(\|\widetilde{\phi}\|+R) R,
$$

so that $\|W(\phi)-\widetilde{\phi}\| \leq R$, provided that the condition (14) holds. Therefore, we obtain $W: \overline{B(\widetilde{\phi}, R)} \rightarrow \overline{B(\widetilde{\phi}, R)}$.

Next, it is easy to prove that $\left\|W^{\prime}(\phi)\right\|<1$ with $W^{\prime}$ defined in (13) if the condition (14) holds.

After that, by the restricted fixed point theorem applied to the operator $W$, we complete the proof of item (a).

Item (b) follows in an analogous way to item (a) without more than taking into account now that

$$
\|\mathcal{H}(\phi)\| \leq\|\mathcal{H}(\phi)-\mathcal{H}(\widetilde{\phi})\|+\|\mathcal{H}(\widetilde{\phi})\| \leq\|\mathcal{H}(\widetilde{\phi})\|+\left(\left\|\mathcal{H}^{\prime}(\widetilde{\phi})\right\|+\omega_{2}(R)\right) R
$$

Next, we present a new result of uniqueness of the fixed point under the conditions (11) or (12), where a technique of functional analysis is used.

Theorem 5. Let $\widetilde{\phi} \in \mathcal{C}[a, b]$.

(a) Under the hypothesis (a) of Theorem 4, suppose that the real equation in $z$ given by

$$
|1-k|+\frac{|k||\lambda| M}{r-R}\left(g_{1}(\|\widetilde{\phi}\|+z)-g_{1}(\|\widetilde{\phi}\|+R)\right)=1,
$$

where $g_{1}(z)=\int_{0}^{z} \omega_{1}(u) d u$, has at least one positive real solution $r$ such that $r>R$. 
(b) Under the hypothesis (b) of Theorem 4, suppose that the real equation in $z$ given by

$$
|1-k|+|k||\lambda| M\left(\left\|\mathcal{H}^{\prime}(\widetilde{\phi})\right\|+\frac{g_{2}(z)-g_{2}(R)}{z-R}\right)=1,
$$

where $g_{2}(z)=\int_{0}^{z} \omega_{2}(u) d u$, has at least one positive real solution $r$ such that $r>R$.

Then, the fixed point of $W$ is unique in $B(\widetilde{\phi}, r)$.

Proof. Suppose that $\phi^{*}$ is a fixed point of $W$ in $\overline{B(\widetilde{\phi}, R)}$ and there exists another fixed point $\varphi^{*} \in B(\widetilde{\phi}, r)$, with $r>R$. Now, from the approximation

$$
\begin{gathered}
\varphi^{*}-\phi^{*}=W\left(\varphi^{*}\right)-W\left(\phi^{*}\right)=\int_{\phi^{*}}^{\varphi^{*}} W^{\prime}(x) d x \\
=\int_{0}^{1} W^{\prime}\left(\phi^{*}+\tau\left(\varphi^{*}-\phi^{*}\right)\right) d \tau\left(\varphi^{*}-\phi^{*}\right),
\end{gathered}
$$

it follows

$$
\int_{0}^{1} A \mathcal{G}^{\prime}\left(\phi^{*}+\tau\left(\varphi^{*}-\phi^{*}\right)\right) d \tau\left(\varphi^{*}-\phi^{*}\right)=0
$$

so that $\varphi^{*}=\phi^{*}$ if the operator $P=\int_{0}^{1} A \mathcal{G}^{\prime}\left(\phi^{*}+\tau\left(\varphi^{*}-\phi^{*}\right)\right) d \tau$ is invertible.

For the last, we prove equivalently that there exists the operator $P^{-1}$. So, from

$$
\begin{aligned}
(I-P) \zeta(x) & =\zeta(x)-\int_{0}^{1} A \mathcal{G}^{\prime}\left(\phi^{*}+\tau\left(\varphi^{*}-\phi^{*}\right)\right) \zeta(x) d \tau \\
& =(1-k) \zeta(x)+\lambda k \int_{0}^{1} \int_{a}^{b} \mathcal{N}(x, t)\left[\mathcal{H}^{\prime}\left(\phi^{*}+\tau\left(\varphi^{*}-\phi^{*}\right)\right) \zeta\right](t) d t d \tau,
\end{aligned}
$$

we have

$$
\|I-P\| \leq|1-k|+|k||\lambda| M \int_{0}^{1}\left\|\mathcal{H}^{\prime}\left(\phi^{*}+\tau\left(\varphi^{*}-\phi^{*}\right)\right)\right\| d \tau .
$$

Now, for item (a) and taking into account (16), it follows

$$
\begin{aligned}
\|I-P\| & \leq|1-k|+|k||\lambda| M \int_{0}^{1} \omega_{1}\left(\phi^{*}+\tau\left(\varphi^{*}-\phi^{*}\right)\right) d \tau \\
& <|1-k|+|k||\lambda| M \int_{0}^{1} \omega_{1}(\|\widetilde{\phi}\|+R+\tau(r-R)) d \tau \\
& =|1-k|+\frac{|k \| \lambda| M}{r-R} \int_{\|\widetilde{\phi}\|+R}^{\|\widetilde{\phi}\|+r} \omega_{1}(t) d t \\
& =1
\end{aligned}
$$

and, by the Banach lemma on invertible operators, we obtain that the operator $P^{-1}$ exists. 
Table 3. Radii of the balls of location and separation of solutions of (6) for different values of $k$ and according to Theorem 4 (a)

\begin{tabular}{lll}
\hline$k$ & $R_{1}$ & $R_{2}$ \\
\hline 0.2 & $0.1801 \ldots$ & $2.7747 \ldots$ \\
0.4 & $0.1801 \ldots$ & $2.7747 \ldots$ \\
0.6 & $0.1801 \ldots$ & $2.7747 \ldots$ \\
0.8 & $0.1801 \ldots$ & $2.7747 \ldots$ \\
1.0 & $0.1801 \ldots$ & $2.7747 \ldots$ \\
1.2 & $0.4064 \ldots$ & $1.2301 \ldots$ \\
\hline
\end{tabular}

For item (b), we provide as for item (a) and taking into account (16), we have

$$
\begin{aligned}
\|I-P\| & \leq|1-k|+|k||\lambda| M \int_{0}^{1}\left(\left\|\mathcal{H}^{\prime}(\widetilde{\phi})\right\|+\omega_{2}\left(\left\|\phi^{*}+\tau\left(\varphi^{*}-\phi^{*}\right)-\widetilde{\phi}\right\|\right)\right) d \tau \\
& <|1-k|+|k||\lambda| M \int_{0}^{1}\left(\left\|\mathcal{H}^{\prime}(\widetilde{\phi})\right\|+\omega_{2}(R+\tau(r-R))\right) d \tau \\
& =|1-k|+|k||\lambda| M\left(\left\|\mathcal{H}^{\prime}(\widetilde{\phi})\right\|+\frac{1}{r-R} \int_{R}^{r} \omega_{2}(t) d t\right) \\
& =1
\end{aligned}
$$

and, by the Banach lemma on invertible operators, we obtain that the operator $P^{-1}$ exists.

Note that the uniqueness of the fixed point $\phi^{*}$ in $\overline{B(\widetilde{\phi}, R)}$ follows from (17) and (18) with $r=R$, since $\|I-P\|<1$ from (14) and (15), respectively, and then $P^{-1}$ exists.

After that, we illustrate Theorems 4 and 5 .

Example 6. Consider the integral equation $(6)$ and $\widetilde{\phi}(x)=x^{3}$. Then, $\|\widetilde{\phi}\|=1$ and, since $\mathcal{H}^{\prime}(\phi)=2 \phi$, we have $\omega_{1}(z)=2 z$, for all $z \in \mathbb{R}_{+}$. From item (a) of Theorem 4, we can guarantee the existence of a unique solution of (6) in $\overline{B\left(x^{3}, R\right)}$ with $R$ satisfying (14), which is reduced respectively to

$$
\frac{1}{5 \mathrm{e}}((\mathrm{e}-1)(2 R(R+1)+1)|k|)+(|1-k|-1) R \leq 0
$$

and satisfied for different values of $k$ in $\overline{B\left(x^{3}, R\right)}$ with $R \in\left[R_{1}, R_{2}\right]$.

In Table 3, we see some values of $k$ and the corresponding radii of the balls $\overline{B\left(x^{3}, R\right)}$ where the existence of a unique fixed point is guaranteed from Theorem 4 (a) and, therefore, a solution of (6). Note that the values of $R_{1}$ and $R_{2}$ are the same for all $k \in[0,1]$.

On the other hand, we illustrate Theorem 5 (a) with Table 4, where the values of $R$ are the values of $R_{2}$ obtained from Theorem 4 (a), see Table 3, and the values of $r$ are the real solutions of the corresponding real equation which 
Table 4. Radii of the balls $B\left(x^{3}, r\right)$ from which the domains of uniqueness of solution of equation (6) are obtained

\begin{tabular}{lll}
\hline$k$ & $R$ & $r$ \\
\hline 0.2 & $2.7747 \ldots$ & $5.7296 \ldots$ \\
0.4 & $2.7747 \ldots$ & $5.7296 \ldots$ \\
0.6 & $2.7747 \ldots$ & $5.7296 \ldots$ \\
0.8 & $2.7747 \ldots$ & $5.7296 \ldots$ \\
1.0 & $2.7747 \ldots$ & $5.7296 \ldots$ \\
1.2 & $1.2301 \ldots$ & $2.8668 \ldots$ \\
\hline
\end{tabular}

is given in item (a) of Theorem 5 . As we can see, the domains of uniqueness of solution $\overline{B\left(x^{3}, R\right)}$ are improved by much with Theorem 5 (a).

Notice that $R \in[0.1801 \ldots, 2.7747 \ldots]$ as long as $k \in[0,1]$ and it seems clear that the condition of Theorem 5 (a) is not satisfied from $k>1.2$. Moreover, if $k \in[1,1.2]$, the higher the value of $k$, the smaller the interval in which $R$ moves, so the domains of existence and uniqueness of solution are worse.

We end by noting that exactly the same results are obtained from item (b) of Theorem 4 and item (b) of Theorem 5 .

We can also give a result of uniqueness of solution from the operator $\mathcal{G}$ instead of the operator $W$, so that the new solution uniqueness result is independent of $k$.

Theorem 7. Let $\widetilde{\phi} \in \mathcal{C}[a, b]$.

(a) Under the hypothesis (a) of Theorem 4, suppose that the real equation in z given by

$$
\frac{|\lambda| M}{z-R}\left(g_{1}(\|\widetilde{\phi}\|+z)-g_{1}(\|\widetilde{\phi}\|+R)\right)=1,
$$

where $g_{1}(z)=\int_{0}^{z} \omega_{1}(u) d u$, has at least one positive real solution $\rho$ such that $\rho>R$.

(b) Under the hypothesis (b) of Theorem 4, suppose that the real equation in $z$ given by

$$
|\lambda| M\left(\left\|\mathcal{H}^{\prime}(\tilde{\phi})\right\|+\frac{g_{2}(z)-g_{2}(R)}{z-R}\right)=1,
$$

where $g_{2}(z)=\int_{0}^{z} \omega_{2}(u) d u$, has at least one positive real solution $\rho$ such that $\rho>R$.

Then, the fixed point of $W$ is unique in $B(\widetilde{\phi}, \rho)$.

Proof. As a fixed point of $W$ is a solution $\phi^{*}$ of the operator (2), we then suppose that $\phi^{*}$ is a solution of $\mathcal{G}$ in $\overline{B(\widetilde{\phi}, R)}$ and there exists another solution 
$\varphi^{*} \in B(\widetilde{\phi}, \rho)$, with $\rho>R$. Now, from the approximation

$$
\begin{aligned}
0 & =\mathcal{G}\left(\varphi^{*}\right)-\mathcal{G}\left(\phi^{*}\right)=\int_{\phi^{*}}^{\varphi^{*}} \mathcal{G}^{\prime}(x) d x=\int_{0}^{1} \mathcal{G}^{\prime}\left(\phi^{*}+\tau\left(\varphi^{*}-\phi^{*}\right)\right) d \tau\left(\varphi^{*}-\phi^{*}\right) \\
& =Q\left(\varphi^{*}-\phi^{*}\right),
\end{aligned}
$$

we have that $\varphi^{*}=\phi^{*}$ if the operator $Q=\int_{0}^{1} \mathcal{G}^{\prime}\left(\phi^{*}+\tau\left(\varphi^{*}-\phi^{*}\right)\right) d \tau$ is invertible.

For the last, we prove equivalently that there exists the operator $Q^{-1}$. So, from

$$
\begin{aligned}
{[(I-Q) \zeta](x) } & =\left[\left(\int_{0}^{1}\left(I-\mathcal{G}^{\prime}\left(\phi^{*}+\tau\left(\varphi^{*}-\phi^{*}\right)\right)\right) d \tau\right) \zeta\right](x) \\
& =\lambda \int_{0}^{1}\left(\int_{a}^{b} \mathcal{N}(s, t)\left[\mathcal{H}^{\prime}\left(\phi^{*}+\tau\left(\varphi^{*}-\phi^{*}\right)\right) \zeta\right](t) d t\right) d \tau
\end{aligned}
$$

it follows

$$
\|I-Q\| \leq|\lambda| M \int_{0}^{1}\left\|\mathcal{H}^{\prime}\left(\phi^{*}+\tau\left(\varphi^{*}-\phi^{*}\right)\right)\right\| d \tau .
$$

Now, for item (a) and taking into account (19), we have

$$
\begin{aligned}
\|I-Q\| & \leq|\lambda| M \int_{0}^{1} \omega_{1}\left(\left\|\phi^{*}+\tau\left(\varphi^{*}-\phi^{*}\right)\right\|\right) d \tau \\
& <|\lambda| M \int_{0}^{1} \omega_{1}(\|\widetilde{\phi}\|+R+\tau(\rho-R)) d \tau \\
& =\frac{|\lambda| M}{\rho-R} \int_{\|\widetilde{\phi}\|+R}^{\|\widetilde{\phi}\|+\rho} \omega_{1}(z) d z \\
& =1
\end{aligned}
$$

and, by the Banach lemma on invertible operators, we obtain that the operator $Q^{-1}$ exists. we have

For item (b), we provide as for item (a) and taking into account (19),

$$
\begin{aligned}
\|I-Q\| & \leq|\lambda| M \int_{0}^{1}\left(\left\|\mathcal{H}^{\prime}(\widetilde{\phi})+\omega_{2}\left(\left\|\phi^{*}+\tau\left(\varphi^{*}-\phi^{*}\right)-\widetilde{\phi}\right\|\right)\right\|\right) d \tau \\
& <|\lambda| M \int_{0}^{1}\left(\left\|\mathcal{H}^{\prime}(\widetilde{\phi})\right\|+\left(\omega_{2}(R+\tau(\rho-R))\right) d \tau\right. \\
& =|\lambda| M\left(\left\|\mathcal{H}^{\prime}(\widetilde{\phi})\right\|+\frac{1}{\rho-R} \int_{R}^{\rho} \omega_{2}(z) d z\right) \\
& =1
\end{aligned}
$$

and, by the Banach lemma on invertible operators, we obtain that the operator $Q^{-1}$ exists.

Note that the uniqueness of the fixed point $\phi^{*}$ in $\overline{B(\widetilde{\phi}, R)}$ follows from (17) with $\rho=R$, provided that

$$
|\lambda| M \omega_{1}(\|\widetilde{\phi}\|+R)<1,
$$


and from (18) with $\rho=R$, provided that

$$
|\lambda| M\left(\left\|\mathcal{H}^{\prime}(\widetilde{\phi})\right\|+\omega_{2}(R)\right)<1,
$$

since $Q^{-1}$ exists in both cases.

Remark 8 . Observe that the uniqueness of solution established by Theorem 7 does not depend on the value of $k$.

Example 9. If we consider the integral equation (6) again, we see that the radius of the ball of uniqueness of solution is $\rho=5.5034 \ldots$ in both cases (item (a) and item (b) of Theorem 7) for $R=1.2301 \ldots$ when $k=1.2$, so that the new domain of uniqueness of solution, $\overline{B\left(x^{3}, 5.5034 \ldots\right)}$, greatly improves the previous ones. On the other hand, if $k \in[0,1]$, the radius of the ball of uniqueness is always the same, $5.7296 \ldots$, which coincides with that obtained from Theorem 5 .

\section{Global Convergence from Auxiliary Functions}

In this section, we present an alternative technique to the previous one, that was based on the restricted fixed point theorem, which also allows us to obtain domains of global convergence, $\overline{B(\widetilde{\phi}, R)}$ with $\widetilde{\phi} \in \mathcal{C}[a, b]$, for the iterative method (3), using auxiliary functions, so that we can locate solutions of (1). This technique was first developed for Newton's method in [10]. For this, we consider the following conditions [5]:

(C1) There exists a constant $\alpha \geq 0$ such that $\|\mathcal{H}(\phi)-\mathcal{H}(\varphi)\| \leq$ $\alpha\|\phi-\varphi\|$, for $\phi, \varphi \in \mathcal{C}[a, b]$.

(C2) There exists a constant $\delta \geq 0$ such that $\left\|\mathcal{H}^{\prime}(\phi)-\mathcal{H}^{\prime}(\varphi)\right\| \leq$ $\delta\|\phi-\varphi\|$, for $\phi, \varphi \in \mathcal{C}[a, b]$.

After that, we present a property that we use later.

Lemma 10. Suppose condition (C2). Then,

$$
\begin{aligned}
\left\|A \mathcal{G}\left(\phi_{n}\right)\right\| \leq & |k-1|\left\|\phi_{n}-\phi_{n-1}\right\|+\left|k\|\lambda \mid M\| \mathcal{H}^{\prime}\left(\phi_{n-1}\right)\|\| \phi_{n}-\phi_{n-1} \|\right. \\
& +\frac{1}{2}|k||\lambda| M \delta\left\|\phi_{n}-\phi_{n-1}\right\|^{2} .
\end{aligned}
$$

Proof. From Taylor's series

$$
\mathcal{G}\left(\phi_{n}\right)=\mathcal{G}\left(\phi_{n-1}\right)+\mathcal{G}^{\prime}\left(\phi_{n-1}\right)\left(\phi_{n}-\phi_{n-1}\right)+\int_{\phi_{n-1}}^{\phi_{n}}\left(\mathcal{G}^{\prime}(\xi)-\mathcal{G}^{\prime}\left(\phi_{n-1}\right)\right) d \xi
$$

we have

$$
\begin{aligned}
A \mathcal{G}\left(\phi_{n}\right)= & (k-1)\left(\phi_{n}-\phi_{n-1}\right)-k \lambda \int_{a}^{b} \mathcal{N}(x, t)\left[\mathcal{H}^{\prime}\left(\phi_{n-1}\right)\left(\phi_{n}-\phi_{n-1}\right)\right](t) d t \\
& +A \int_{0}^{1}\left(\mathcal{G}^{\prime}\left(\phi_{n-1}+\tau\left(\phi_{n}-\phi_{n-1}\right)\right)-\mathcal{G}^{\prime}\left(\phi_{n-1}\right)\right)\left(\phi_{n}-\phi_{n-1}\right) d \tau
\end{aligned}
$$

so that (22) follows immediately. 
From Lemma 10, we analyze the first iteration of the iterative method (3), what leads us to the convergence of the method.

If $\phi_{0} \in \overline{B(\widetilde{\phi}, R)}$, then

$$
\begin{aligned}
\left\|\phi_{1}-\phi_{0}\right\| & =\left\|A \mathcal{G}\left(\phi_{0}\right)\right\| \\
& =\left\|k \phi_{0}-k f-k \lambda \int_{a}^{b} \mathcal{N}(x, t) \mathcal{H}\left(\phi_{0}\right)(t) d t\right\| \\
& \leq|k|(R+\|\widetilde{\phi}-f\|)+|k \| \lambda| M\left(\|\mathcal{H}(\widetilde{\phi})\|+\alpha\left\|\phi_{0}-\widetilde{\phi}\right\|\right) \\
& \leq|k|(\|\widetilde{\phi}-f\|+|\lambda| M\|\mathcal{H}(\widetilde{\phi})\|+(1+|\lambda| M \alpha) R) \\
& =\varepsilon,
\end{aligned}
$$

provided that the condition (C1) holds, and

$$
\begin{aligned}
\left\|\phi_{1}-\widetilde{\phi}\right\| & =\left\|\phi_{0}-A \mathcal{G}\left(\phi_{0}\right)-\widetilde{\phi}\right\| \\
& =\left\|\phi_{0}-k \phi_{0}+k f+k \lambda \int_{a}^{b} \mathcal{N}(x, t) \mathcal{H}\left(\phi_{0}\right)(t) d t-\widetilde{\phi}\right\| \\
& \leq|1-k|\left\|\phi_{0}-\widetilde{\phi}\right\|+|k|\|f-\widetilde{\phi}\|+|k \| \lambda| M\left(\|\mathcal{H}(\widetilde{\phi})\|+\alpha\left\|\phi_{0}-\widetilde{\phi}\right\|\right) \\
& \leq|k|(\|f-\widetilde{\phi}\|+|\lambda| M\|\mathcal{H}(\widetilde{\phi})\|)+(|1-k|+|k||\lambda| M \alpha) R,
\end{aligned}
$$

so that $\phi_{1} \in \overline{B(\widetilde{\phi}, R)}$, provided that

$$
|k|(\|f-\widetilde{\phi}\|+|\lambda| M\|\mathcal{H}(\widetilde{\phi})\|)+(|1-k|+|k||\lambda| M \alpha) R \leq R .
$$

Now, if we suppose that

$$
\begin{aligned}
& \left\|\phi_{n}-\phi_{n-1}\right\|<\gamma\left\|\phi_{n-1}-\phi_{n-2}\right\| \\
& \left\|\phi_{n}-\widetilde{\phi}\right\| \leq|k|(\|f-\widetilde{\phi}\|+|\lambda| M\|\mathcal{H}(\widetilde{\phi})\|)+(|1-k|+|k \| \lambda| M \alpha) R \leq R
\end{aligned}
$$

where

$$
\gamma=|1-k|+|k||\lambda| M(\|\mathcal{H}(\widetilde{\phi})\|+\alpha R)+\frac{1}{2}|k||\lambda| M \alpha \varepsilon
$$

for all $n \geq 2$, and provided that condition (23) holds, it follows in the same way that

$$
\left\|\phi_{n+1}-\phi_{n}\right\|<\gamma\left\|\phi_{n}-\phi_{n-1}\right\| \quad \text { and } \quad\left\|\phi_{n+1}-\tilde{\phi}\right\| \leq R,
$$

so that (24) and (25) are true for all positive integers $n$ by mathematical induction.

Next, we can establish the following result.

Theorem 11. Suppose conditions (C1)-(C2) and consider $R>0$ such that (23) and $\gamma<1$ hold. Then, the iterative method (3) with $k \in(0,2)$ is well-defined and converges starting at any $\phi_{0} \in B(\widetilde{\phi}, R)$ to a solution $\phi^{*}$ of $\mathcal{G}(\phi)=0$ in $\overline{B(\widetilde{\phi}, R)}$. 
Table 5. Radii of the balls of location of solutions of (6) for different values of $k$ and according to Theorem 11

\begin{tabular}{llll}
\hline$k$ & $R$ & $k$ & $R$ \\
\hline 0.2 & $1.0333 \ldots$ & 1.0 & $0.9678 \ldots$ \\
0.4 & $1.1864 \ldots$ & 1.2 & $0.6559 \ldots$ \\
0.6 & $1.1008 \ldots$ & 1.4 & $0.3946 \ldots$ \\
0.8 & $1.0291 \ldots$ & 1.6 & $0.1577 \ldots$ \\
\hline
\end{tabular}

Table 6. Absolute errors for integral equation (6)

\begin{tabular}{ll}
\hline$n$ & $\left\|\phi^{*}-\phi_{n}\right\|$ \\
\hline 0 & $1.2259 \ldots \times 10^{-2}$ \\
1 & $2.7224 \ldots \times 10^{-4}$ \\
2 & $6.2528 \ldots \times 10^{-6}$ \\
3 & $1.4371 \ldots \times 10^{-7}$ \\
4 & $3.3032 \ldots \times 10^{-9}$ \\
5 & $7.5924 \ldots \times 10^{-11}$ \\
6 & $1.7450 \ldots \times 10^{-12}$ \\
7 & $4.0110 \ldots \times 10^{-14}$ \\
8 & $9.2192 \ldots \times 10^{-16}$ \\
\hline
\end{tabular}

Proof. From (24) and $\gamma<1$, we have $\left\|\phi_{n+1}-\phi_{n}\right\|<\left\|\phi_{n}-\phi_{n-1}\right\|$, for all $n \in \mathbb{N}$, so that sequence $\left\{\left\|\phi_{n+1}-\phi_{n}\right\|\right\}$ is strictly decreasing for all $n \in \mathbb{N}$ and, therefore, sequence $\left\{\phi_{n}\right\}$ is convergent. If $\phi^{*}=\lim _{n \rightarrow \infty} \phi_{n}$, then $\mathcal{G}\left(\phi^{*}\right)=0$ by the continuity of $\mathcal{G}$ and $\left\|A \mathcal{G}\left(\phi_{n}\right)\right\| \rightarrow 0$ when $n \rightarrow \infty$, since $k \in(0,2)$.

Example 12. Now, we apply Theorem 11 to the integral equation (6). For this, we consider again $\widetilde{\phi}(x)=x^{3}$, so that $\alpha=2(1+R)$, since $\|\mathcal{H}(\phi)-\mathcal{H}(\varphi)\|=$ $\left\|\phi^{2}-\varphi^{2}\right\|$, for $\phi, \varphi \in \mathcal{C}[0,1]$. Morevoer, $\delta=2$, since $\left\|\mathcal{H}^{\prime}(\phi)-\mathcal{H}^{\prime}(\varphi)\right\|=$ $2\|\phi-\varphi\|$, for $\phi, \varphi \in \mathcal{C}[0,1]$. Furthermore, (23) and $\gamma<1$ are satisfied at the same time by different values of $k \in(0,2)$ in $\overline{B(\widetilde{\phi}, R)}$. In Table 5 we see some values of $k$ and the corresponding radii of the balls $\overline{B(\widetilde{\phi}, R)}$ where the existence of a solution of the integral equation (6) is guaranteed from Theorem 11.

Besides, it seems clear that the conditions of Theorem 11 are not satisfied if $k \in(1.72,2)$. In addition, the higher the value of $k$, the smaller the value of $R$, so that the best location of a solution is when $k$ is the largest possible value. Finally, by Theorem 11, we extend, with respect to the study presented in Sect. 3, the application of the iterative method (3) to obtain domains of existence of solution for the integral equation (6).

Finally, from $\phi_{0}(x)=x^{3}$, we apply the Whittaker-type method given in $(3)$, with $A(\phi)(x)=k \phi(x)$ and $k=\frac{1}{2}$, and obtain the solution $\phi^{*}(x)=$ 
$x^{3}+(0.0122 \ldots) \mathrm{e}^{-x}$ after eight iterations with the stopping criterion $\| \phi_{n}(s)-$ $\phi_{n-1}(s) \|<10^{-16}$. In Table 6 , we show the errors $\left\|\phi^{*}(s)-\phi_{n}(s)\right\|$. Similar results are obtained for any other value of $k \in(0,2)$.

\section{Conclusions}

The fixed point theorem is a known result to obtain results of global convergence. But, from the use of this theorem and the previous location of fixed points, it is not always possible to find domains of global convergence, so that they contain fixed points that can be separated from others (for this, see Sect. 2). In addition to not correctly locating a fixed point or separating it from another possible fixed point. In this work, we locate fixed point in balls of the form $\overline{B(\widetilde{\phi}, R)}$, where $\widetilde{\phi}$ is an auxiliary function, which also allows us to separate them from others with great accuracy. For this, we use two techniques: one based on a restricted fixed point theorem and the other on a system of recurrence relations. In both techniques, we use auxiliary functions and illustrate the study with a Whittaker-type iterative method and Fredholm-Nemytskii nonlinear integral equations.

Funding Open Access funding provided thanks to the CRUE-CSIC agreement with Springer Nature.

Open Access. This article is licensed under a Creative Commons Attribution 4.0 International License, which permits use, sharing, adaptation, distribution and reproduction in any medium or format, as long as you give appropriate credit to the original author(s) and the source, provide a link to the Creative Commons licence, and indicate if changes were made. The images or other third party material in this article are included in the article's Creative Commons licence, unless indicated otherwise in a credit line to the material. If material is not included in the article's Creative Commons licence and your intended use is not permitted by statutory regulation or exceeds the permitted use, you will need to obtain permission directly from the copyright holder. To view a copy of this licence, visit http:// creativecommons.org/licenses/by/4.0/.

Publisher's Note Springer Nature remains neutral with regard to jurisdictional claims in published maps and institutional affiliations.

\section{References}

[1] Amat, S., Busquier, S., Plaza, S.: Review of some iterative root-finding methods from a dynamical point of view. Sci. Ser. A Math. Sci. (N.S.) 10, 3-35 (2004)

[2] Argyros, I.K.: On a class of nonlinear integral equations arising in neutron transport. Aequationes Math. 36(1), 99-111 (1988)

[3] Argyros, I.K.: Approximating the fixed points of some nonlinear operator equations. Math. Slovaca 38(4), 409-417 (1988) 
[4] Argyros, I.K., George, S.: Extending the applicability of the super-Halley-like method using $\omega$-continuous derivatives and restricted convergence domains. Ann. Math. Sil. 33(1), 21-40 (2019)

[5] Argyros, I.K., Regmi, S.: Undergraduate research at Cameron University on iterative procedures in Banach and other spaces. Nova Science Publisher, New York (2019)

[6] El-Sayed, A.M.A., Hashem, H.H.G., Ziada, E.A.A.: Picard and Adomian methods for quadratic integral equation. Comput. Appl. Math. 29(3), 447-463 (2010)

[7] Ezquerro, J.A., Hernández, M.A.: On an application of Newton's method to nonlinear operators with $\omega$-conditioned second derivative. BIT 42, 519-530 (2002)

[8] Ezquerro, J.A., Hernández, M.A.: Generalized differentiability conditions for Newton's method. IMA J. Numer. Anal. 22(2), 187-205 (2002)

[9] Ezquerro, J.A., Hernández-Verón, M.A.: Newton's method: an updated approach of Kantorovich's theory. Frontiers in Mathematics. Birkhäuser/Springer, Cham (2017)

[10] Ezquerro, J.A., Hernández-Verón, M.A.: Domains of global convergence for Newton's method from auxiliary points. Appl. Math. Lett. 85, 48-56 (2018)

[11] Ezquerro, J.A., Hernández-Verón, M.A.: How to obtain global convergence domains via Newton's method for nonlinear integral equations. Mathematics 7, 553 (2019)

[12] Ezquerro, J.A., Hernández-Verón, M.A.: Domains of global convergence for a type of nonlinear Fredholm-Nemytskii integral equations. Appl. Numer. Math. 146, 452-468 (2019)

[13] Ezquerro, J.A., Hernández-Verón, M.A.: Mild differentiability conditions for Newton's method in Banach spaces. Frontiers in Mathematics. Birkhäuser/Springer, Cham (2020)

[14] Hernández, M.A.: An acceleration procedure of the Whittaker method by means of convexity. Zb. Rad. Prirod.-Mat. Fak. Ser. Mat. 20(1), 27-38 (1990)

[15] Rashidinia, J., Zarebnia, M.: Convergence of approximate solution of system of Fredholm integral equations. J. Math. Anal. Appl. 333(2), 1216-1227 (2007)

[16] Regmi, S.: Optimized iterative methods with applications in diverse disciplines. Nova Science Publisher, New York (2021)

[17] Shizgal, B.: Integral equations in the kinetic theory of gases and related topics. Spectral methods in chemistry and physics. Springer, Dordrecht (2015)

[18] Zeynalli, S.M., Abbasova, S.V., Gurbanova, A.G.: Application of the Picard method of sequential integration in differential inequalities. Adv. Math. Model. Appl. 3(2), 164-167 (2018)

\author{
J. A. Ezquerro and M. A. Hernández-Verón \\ Department of Mathematics and Computation \\ University of La Rioja \\ Calle Madre de Dios, 53 \\ 26006 Logroño La Rioja \\ Spain \\ e-mail: jezquer@unirioja.es
}


M. A. Hernández-Verón

e-mail: mahernan@unirioja.es

Received: January 19, 2021.

Revised: March 11, 2021.

Accepted: December 1, 2021. 